\title{
Augmented Renal Clearance in COVID-19
}

\author{
Ahmet Murt Mevlut Tamer Dincer Cebrail Karaca \\ Nephrology Unit, Cerrahpasa Medical Faculty, Istanbul University-Cerrahpasa, Istanbul, Turkey
}

Dear Editor,

The concept of augmented renal clearance (ARC) has been previously proposed in critically ill patients [1]. Vasodilatation and the hyperdynamic state in systemic inflammation result in high cardiac output, and renal perfusion may be enhanced with an increase in glomerular filtration rate (GFR). It is now very well known that the hyper-inflammation state in the context of cytokine release syndrome might be a component of the clinical picture of COVID-19. Aggressive fluid resuscitation and certain vasoactive drugs in the management of the systemic inflammatory response may also contribute to an increase in renal perfusion, which eventually increases GFR.

Cr levels tended to decrease in 13 of our patients with COVID-19. ARC could be defined in 5 patients who had their estimated GFR reaching more than $130 \mathrm{~mL} / \mathrm{min} / 1.73$ $\mathrm{m}^{2}$. Their mean age was $42.6 \pm 21.8$ years and there was a male predominance $(80 \%)$. The patients had an average basal Cr of $0.81 \pm 0.12 \mathrm{mg} / \mathrm{dL}$, their Cr levels dropped as low as $0.33 \pm 0.07 \mathrm{mg} / \mathrm{dL}$, and their estimated GFR increased to $154.4 \pm 19.8 \mathrm{~mL} / \mathrm{min} / 1.73 \mathrm{~m}^{2}$. The median day to observe ARC was the 13th day of patients' hospital admission [range: $3-46$ days]. Patients spent $5.0 \pm 1.0$ days with their ARC functions and the average length of hospital stay of the patients was $33.6 \pm 25.6$ days.

ARC was found to coincide with an increase in inflammatory markers. The day when ARC first observed was strongly correlated with the day of peak ferritin, the day of peak C-reactive protein and the day of peak D-dimer (Pearson's $r=0.82$ [ $p=0.08$ ], 0.92 [ $p=0.03$ ], and 0.88 $[p=0.04]$, respectively). Such high correlations indicate the inflammatory response as the pathophysiologic background of ARC in patients with COVID-19. These patients also had high intensive care admission and mortality rates at 80 and $60 \%$, respectively.

It was previously proposed that GFR might increase with certain stimuli and the capacity of kidneys to increase GFR was called the renal functional reserve [2]. This concept may explain the protection of a certain level of GFR after kidney injuries. However, as seen in our cohort, it may be a surrogate marker of a systemic inflammatory response.

In addition to being a marker to show the hyper-inflammatory state, ARC may also have therapeutic failure consequences with an increase in renal clearance of drugs [3]. Dosing of certain renally eliminated drugs should be evaluated in conjunction with GFR calculations. It should be noted that Chronic Kidney Disease Epidemiology Collaboration (CKD-EPI) and Modification of Diet in Renal Disease (MDRD) formulas may underestimate GFR. Although 24-h urine studies would give the most accurate results, 8 -h studies will also be adequate for frequent calculations [4]. karger@karger.com

www.karger.com/nef

Karger!

(n) (c) 2021 S. Karger AG, Basel
Nephrology Unit, Internal Medicine Department, Cerrahpasa Medical Faculty Istanbul University-Cerrahpasa

Istanbul 34098 (Turkey)

ahmet.murt@istanbul.edu.tr 
Similar to our cohort, who were younger than general inpatients with COVID-19, younger age was previously defined as a contributor in the development of ARC [5]. In addition to acute kidney injury, which has been shown as a poor prognostic factor in COVID-19, physicians should also take care about the tendency for a decrease in Cr levels of their patients with COVID-19 as the latter might indicate an inflammatory response.

\section{Conflict of Interest Statement}

The authors declare no conflicts of interest.

\section{Funding Sources}

The authors did not receive any funding.

\section{Author Contributions}

Ahmet Murt conceptualized the idea, collected and analyzed the data, performed the literature search, and wrote the first draft of the text. Mevlut Tamer Dincer and Cebrail Karaca helped in the collection of the data. All authors commented on the previous versions of the manuscript and approved the final version.

\section{References}

1 Bilbao-Meseguer I, Rodríguez-Gascón A, Barrasa H, Isla A, Solinís MÁ. Augmented renal clearance in critically ill patients: a systematic review. Clin Pharmacokinet. 2018 Sep; 57(9):1107-21.

2 Sharma A, Mucino MJ, Ronco C. Renal functional reserve and renal recovery after acute kidney injury. Nephron Clin Pract. 2014; 127(1-4):94-100.
3 Mahmoud SH, Shen C. Augmented renal clearance in critical illness: an important consideration in drug dosing. Pharmaceutics. 2017 Sep 16;9(3):36

4 Chen IH, Nicolau DP. Augmented renal clearance and how to augment antibiotic dosing. Antibiotics. 2020 Jul;9(7):393.

5 Nei AM, Kashani KB, Dierkhising R, Barreto EF. Predictors of augmented renal clearance in a heterogeneous ICU population as defined by creatinine and cystatin C. Nephron. 2020; 144(7):313-20. 